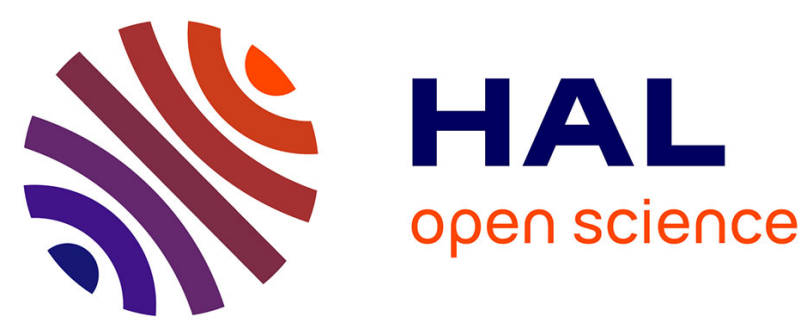

\title{
Performance and implementation of UFA : a SIP-based ultra flat mobile network architecture
}

\author{
Khadija Daoud, Philippe Herbelin, Karine Guillouard, Noel Crespi
}

\section{To cite this version:}

Khadija Daoud, Philippe Herbelin, Karine Guillouard, Noel Crespi. Performance and implementation of UFA : a SIP-based ultra flat mobile network architecture. PIMRC 2009: IEEE International Symposium on Personal, Indoor and Mobile Radio Communications, Sep 2009, Tokyo, Japan. pp.793 - 797, 10.1109/PIMRC.2009.5450117 . hal-01368589

\section{HAL Id: hal-01368589 \\ https://hal.science/hal-01368589}

Submitted on 19 Sep 2016

HAL is a multi-disciplinary open access archive for the deposit and dissemination of scientific research documents, whether they are published or not. The documents may come from teaching and research institutions in France or abroad, or from public or private research centers.
L'archive ouverte pluridisciplinaire HAL, est destinée au dépôt et à la diffusion de documents scientifiques de niveau recherche, publiés ou non, émanant des établissements d'enseignement et de recherche français ou étrangers, des laboratoires publics ou privés. 


\section{Performance and Implementation of UFA: a SIP- based Ultra Flat Mobile Network Architecture}

\author{
Khadija Daoud, Philippe Herbelin, Karine Guillouard \\ Orange Labs, Issy Les Moulineaux, France \\ \{first name.last name\} @ orange-ftgroup.com
} network architectures as well as IMS layer will not be able to handle the increasing number of mobile users consuming high bitrate services. An Ultra Flat Architecture (UFA), based on SIP bitrate services. An Ultra Flat Architecture (UFA), based on SIP
and designed with the primary goal of being scalable, has been introduced in a previous paper. In this paper, the limitations of current 3GPP architectures are discussed and detailed explanations of UFA interest and procedures are provided. UFA is entirely controlled by the operator and integrates $\mathrm{QOS}$ in its establishment and mobility procedures. To prove its concept and establishment and mobility procedures. To prove its concept and
assess the performance of its mobility procedure, UFA is implemented on a testbed. UFA HO delay measured at the application level is $100 \mathrm{~ms}$ in average; it is $\mathbf{1 2}$ times smaller than in a classic SIP-based mobility scheme.

Keywords-component: flat architecture, UFA, SIP, IMS, QoS, HO

\section{INTRODUCTION}

In recent years, advances in mobile device capabilities and radio technologies have been quite remarkable. This has enabled mobile subscribers to access from mobile networks the same variety of broadband services experienced on fixed networks. Same as for fixed networks, it is expected that a high number of mobile users will consume these services; causing a high traffic volume and scalability issues. To face this problem, new network architectures have been designed with fewer nodes; they are called flat architectures. Despite this effort, scalability issues are still not entirely solved since these architectures remain centralised. IMS [1], the service control layer on top of fixed and mobile networks, is also centralised and is subject to scalability issues. We previously introduced in [2] a new flat architecture named UFA (Ultra Flat Architecture) that aims at solving simultaneously mobile networks and IMS scalability issues. UFA is considered as an ultimate step towards flat architectures, its principle is to distribute into the Base Stations (BSs), the single nodes of this architecture, data forwarding plane functions and control plane functions including IMS ones. In [2] we studied the impact of anchor-based mobility management protocols on network scalability and defined the principles of UFA establishment and mobility procedures, ending with an analytical evaluation of handover (HO) performance. In this paper, we consider the 3 GPP mobile architectures and the IMS network and show their limitations when faced with a high number of users. We based our analysis on the location of the first IP router seen by the Mobile Node (MN) and the number of user related contexts maintained by each of IMS and mobile network nodes. Regarding UFA, we thoroughly describe the mobility procedure taking into account the case of mono and mult interfaced MNs. We prove the UFA concept through testbed-

\author{
Noël Crespi \\ Institut Telecom, telecom SudParis, Evry, France \\ noel.crespi@it-sudparis.eu
}

based implementation and analyse the measured performance results, which was not performed in [2].

II. MOBILE NETWORKS AND IMS SCALABILITY ISSUES

Many criteria can be used to measure network scalability. They are: the location of the first IP router seen by the MN, the number of intermediate nodes on the data forwarding path between the MN and the first IP router, the number of nodes on the signalling path, the number of network interfaces, etc. The first IP router is responsible for allocating an IP address to the MN. It is considered as the first IP hop for the MN and anchors traffic between the internet and MNs and even between MNs attached to the same network

1) UMTS:- UMTS [3] is a 3GPP mobile network composed of four specific nodes that are from top to down: the GGSN, the SGSN, the RNC and the NodeBs. The GGSN is the first IP router. The SGSN performs user authentication, stores for each user ciphering keys, and forwards data to the $\mathrm{MN}$ according to the routing and QoS (Quality of Service) information built during bearer establishment. The RNC is the intelligent part of the radio access network (UTRAN) and performs data ciphering, data compression as well as radio resource allocation. All of these four nodes maintain a per-user context and are on the forwarding data path, which makes them sensitive to the user number and traffic increase. To reduce scalability issues incurred by this hierarchical and centralised design, 3GPP has introduced in [3] a new option called "Direct Tunnel" where data on the forwarding plane does not need to pass through the SGSN; it is directly tunnelled from the GGSN to the RNC. Flat HSPA+ [4] is another evolution of UMTS that has been introduced to enhance UMTS scalability by removing the RNC and distributing its functions in the NodeBs.

2) EPS: EPS (Evolved Packet System) or SAE/LTE [5] has been defined in Release 8 of 3GPP, it provides an IP-based packet core network (SAE, (System Architecture Evolution)) that enables users to access operator's 3GPP IP services, whatever the access network (3GPP and non-3GPP), and to ensure mobility between these access networks. In this framework, 3GPP has also defined an evolved radio access network called LTE (Long Term Evolution) [6] that offers high radio bitrates. As shown in Figure 1, SAE architecture mainly introduces three new entities: the P-GW (Packet Data Network Gateway), the S-GW (Serving Gateway) and the MME (Mobility Management Entity). The P-GW is the first IP route for all users, it uses anchor-based mobility management protocols (e.g. PMIP [7]) to manage the mobility of users between 3GPP access systems and non-3GPP access systems,
Mis en forme : Espace Avant : $6 \mathrm{pt}$

Mis en forme : Espace Avant : $6 \mathrm{pt}$ 
it can be assimilated to the GGSN in terms of functions. The SGW manages the mobility of users between 3GPP access systems (UTRAN, LTE) and is equivalent to the SGSN data forwarding plane functions. The MME contains the SGSN control plane operations and a few control plane security functions performed by the RNC in UMTS. LTE is considered as a flat access network since it consists only of one node type, the eNodeBs (LTE BSs) containing all the radio intelligence.

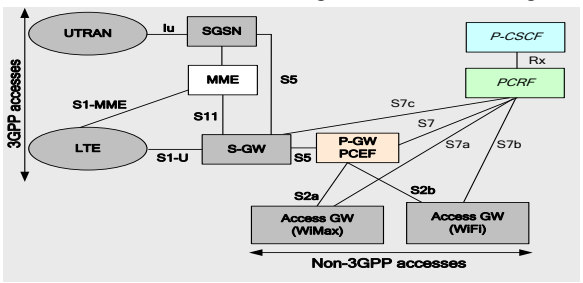

Figure 1. LTE/SAE architecture

3) IMS: IMS (IP Multimedia Subsystem) [1] is defined within $3 \mathrm{GPP}$ as a service framework and as a mean for fixed and mobile network operators to provide the adequate QoS for transported services on the one hand, and perform a servicebased differentiated charging on the other hand. IMS introduces independent network components based on SIP [8]; they are the Proxy Call Service Control function (P-CSCF), the Interrogating CSCF (I-CSCF), and the Serving CSCF (SCSCF). The S-CSCF is located in the home network, it performs user authentication and implements the actual SIP registrar functionality and session control. The P-CSCF acts as a SIP proxy between the MN and the I-CSCF/S-CSCF and is located behind the first IP router of mobile networks. It stores for each terminal accounting and security association data. During IMS authentication procedure, the P-CSCF establishes an IPsec tunnel with the MN to provide integrity and ciphering for the transported SIP signaling. To ensure permanent MN reachability, this tunnel shall be maintained along the MN attachment period, imposing the need of an always-on IP connectivity in the mobile network. The P-CSCF also stores the Service Information $(S I)$ deduced during session establishment. To enforce the QoS corresponding to SI on the mobile network, a new entity called PCRF (Policy and Charging Rules Function) has been introduced by the PCC architecture [9]. The P-CSCF sends SI to the PCRF. The PCRF deduces IP Authorized $Q o S$ and transfers it to the first IP router in the mobile network, called PCEF (Policy and Charging Enforcement Function). The PCEF deduces Access Specific $Q o S$ and ensures that the bearer established on the network level to handle the session traffic does not exceed this QoS. Figure 2 shows the number of contexts maintained in IMS and PCC architectures. Application of PCC architecture to SAE architecture is given in Figure 1. As the mobility protocol (e.g. PMIP) between the P-GW and the access gateways is not a bearer establishment protocol, new interfaces (see S7x in Figure 1) have been added by 3GPP between the PCRF and these gateways; increasing thus scalability risks. IMS and PCC architectures have other limitations regarding signaling cost and QoS management [10]. Indeed, PCC architecture role is limited to the enforcement of a given QoS on the mobile network independently of whether resources are available or not. PCC entities could react to resource availability information in order to adapt the service accordingly; howeve as this information is distributed in all mobile network nodes reporting this information to PCC nodes could not be ensured without consequent modifications on the specified architectures.

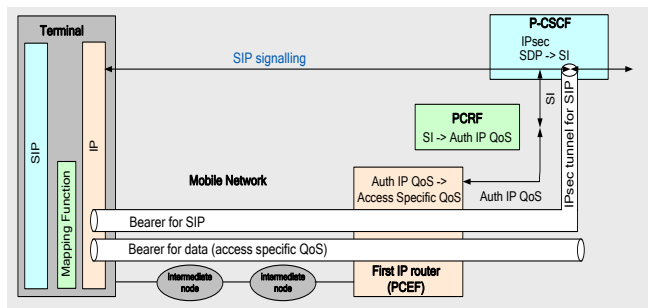

Figure 2. Contexts in IMS and PCC architectures nodes

4) Discussion on mobile networks and IMS scalability: The flattening approach appears to be a solution for the expected scalability risks; however the way it is applied for LTE/SAE and UMTS (HSPA+) does not solve all scalability issues. Even if these architectures remove some of the intermediate nodes, the first IP router is still centralised and manages a high number of users. As the PCRF and the P-CSCF entities are both located behind this first IP router they inherit the same kind of scalability issues. Duplicating the first IP routers, the PCSCFs and the PCRFs can be envisaged when anticipating more and more subscribers in the future. However, as discussed in [11], load-aware algorithms for the selection of the less loaded node as well as a protocol to update the load of each node shall be deployed. Besides, distributing users on the different nodes can not be performed dynamically as these nodes cannot be changed during the lifetime of an established communication.

\section{UFA ARCHITECTURE}

An end-to-end architecture solution shall be defined to solve scalability issues. UFA has been proposed with this design objective. It distributes the first IP router, the PCRF and the P-CSCF functions in the BSs, now called UFA_GW. We choose to use SIP in the UFA GW as the main protocol for UFA establishment and mobility procedures for the following reasons: 1) SIP is adopted in 3GPP for IMS 2) it can offer to operators the knowledge of the established applications 3 ) it is independent of the radio technology making UFA mobility procedures applicable to inter and intra technology HOs 4) it enables at the same time to establish sessions, manage their mobility and transport their related application description (SDP, Session Description Protocol [12]). In the UFA GW, the SIP layer acts as a B2BUA (Back-To-Back User Agent) in order to control SIP messages and perform their modification according to the operator policies. Thanks to this control and because the UFA GWs simultaneously manage the SIP layer and all the network resource information, adapting applications to resource availability become possible. In this way, establishment/mobility procedure implicitly integrates QoS 
IV. UFA ESTABLISHMENT AND MOBILITY PROCEDURES

When MN wants to initiate a service with a $\mathrm{CN}$ (Corresponding Node), it sends a SIP INVITE message to this $\mathrm{CN}$ to create a SIP session. The B2BUA in the Source UFA_GW (UFA_GW_S) stops this message and creates a second SIP INVITE message towards the CN. This induces the creation of two SIP sessions: one (MN-UFA_GW_S) between MN and UFA_GW_S and the other one (UFA_GW_S-CN) between UFA_GW_S and CN; each session is identified by a call ID. The B2BUA binds the two SIP sessions related to the established service. For a SIP message received from MN (resp. CN), From header, To header and MN (resp. CN) IP address in the SDP $c$ field are copied in the corresponding SIP message sent to $\mathrm{CN}$ (resp. MN). Call ID is changed, $\mathrm{MN}$ (resp. $\mathrm{CN}$ ) IP address in the contact header is replaced by UFA_GW_S IP address, and SDP content can be changed. Indeed, during service establishment, after the $\mathrm{MN}$ and the $\mathrm{CN}$ agree on a Requested SDP, the UFA GW S stores the Requested SDP and checks whether it can allocate the resources necessary to satisfy this SDP. If not it proposes another SDP Proposed SDP and sends it to $\mathrm{MN}$ and $\mathrm{CN}$ so that they begin their call with the corresponding application description, more details can be found in [10]. The Requested $S D P$ can be later used by UFA_GW_S to propose a session upgrade when more resources become available.

As shown in Figure 3, when a $\mathrm{HO}$ is anticipated by UFA_GW_S, (1) it sends to a set of candidate GWs a Resource Query Request that includes Requested SDP. (2) Each of these candidates answers according to its resources with a Proposed SDP. UFA_GW_S then selects a target UFA_GW (UFA_GW_T) and begins HO preparation. HO preparation aims at transferring the two SIP sessions (MN - UFA GW S) and (UFA GW $S-C N$ ) from UFA GW $S$ to UFA GW T and pre-determining all the OSI layers configuration required for MN after its HO. The SIP REFER [13] method is not used as it cannot entirely answer to this objective; it is accompanied by the creation of new SIP sessions ((MN-UFA_GW_T) and (UFA_GW_T-CN)) which would necessitate a supplementary mechanism to bind these two new sessions. (3) Therefore, UFA GW S sends to UFA GW T Context Transfer message which includes both SIP sessions description (From, To, Contact, Requested SDP, MN capabilities, etc.). UFA_GW_T allocates an IP address for the MN (Add_IP Addr), checks its uniqueness, confirms the Proposed SDP and (4) includes them with other UFA_GW_T related information in $(A C K)$ message towards UFA_GW_S. $(5,5 \mathrm{~A})$ Based on the received message, UFA GW S builds two SIP re-INVITE messages to send to $\mathrm{MN}$ and $\mathrm{CN}$.

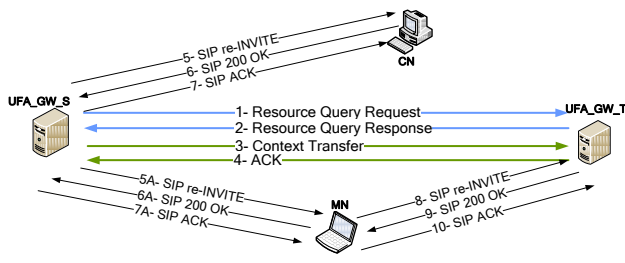

Figure 3.UFA handover procedure
A SIP re-INVITE message enables to update information related to an existing dialog (e.g. Contact header, SDP, $c$ field of SDP). As (UFA_GW_S-CN) and (MN-UFA_GW_S) SIP sessions are transferred from UFA_GW_S to UFA_GW_T, SIP re-INVITE messages towards $\mathrm{CN}$ and $\mathrm{MN}$ have a contact header equal to UFA GW T IP address. They also include Proposed SDP; with a $c$ field for the SIP re-INVITE message towards $\mathrm{CN}$ equal to the new IP address of $\mathrm{MN}$ after its $\mathrm{HO}$ (Add IP Addr). This indicates to $\mathrm{CN}$ to send data to this address. In order to provide better QoS and trigger the MN handover at L2 and L3 using the same messages, two new SIP headers are included in the SIP re-INVITE messages. The firs SIP header is UFA Appli_Config and is sent to $\mathrm{CN}$ and $\mathrm{MN}$. As shown Table 1, it contains $A d d \_I P \_A d d r$ and indicates to the application whether data bi-casting to/from the current $\mathrm{MN}$ IP address and Add IP Addr has to be performed during HO period so that data loss can be minimized. The second header is UFA_Terminal_Config and is only sent to MN to provide the necessary inputs for L2 and L3 HOs execution. UFA_Terminal_Config definition applies to the cases of mono and multi-interfaced MN. As shown in Table 2Table 2, it includes information about UFA_GW_T and indicates to MN the interface (Interface_Name_Target) to attach to UFA_GW_T for the session subject to $\mathrm{HO}$ and indicated by the SIP INVITE Call ID. It also indicates to MN whether the current IP address (Del_IP_Addr) of its current interface (Interface_Name_Source) shall continue to be attached to UFA_GW_S. In case of hard HO, Interface Name Target is equal to Interface_Name_Source. UFA_Terminal_Config concerns the MN network layer configuration; therefore it is duplicated in all SIP re-INVITE messages related to sessions subject to the same network handover (same UFA_GW_T).

Table 1. UFA_Appli_Config header

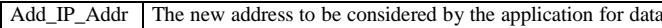
\begin{tabular}{|l|l} 
& transmission \\
\hline Bi-cast $=0$ or 1 & Indicates whether bi-casting shall be performed or not.
\end{tabular}

Bi-cast $=0$ or 1 Indicas whether bi-casing shall be perforned or

\begin{tabular}{|c|c|}
\hline Interface_Name_Target & $\begin{array}{l}\text { The interface to be activated on the MN to } \\
\text { handle the session subject to handover. This } \\
\text { interface will be attached to UFA_GW_T }\end{array}$ \\
\hline UFA_GW_T_MAC_Addr & \multirow[t]{3}{*}{ Used for L2 HO } \\
\hline UFA_GW_T_ESSID & \\
\hline UFA_GW_T_Channel & \\
\hline UFA_GW_T_IP_Addr & \multirow[t]{3}{*}{ Used for IP HO } \\
\hline UFA_GW_T_Netmask & \\
\hline Add_IP_Addr & \\
\hline Interface_Name_Source & $\begin{array}{l}\text { The current MN interface that handles the } \\
\text { session subject to handover }\end{array}$ \\
\hline $\begin{array}{l}\text { Del_IP_Addr } \\
0 \text { or value }\end{array}$ & $\begin{array}{l}\text { Specifies whether the current IP address } \\
\text { (Del_IP_Addr) on the Interface_Name_Source } \\
\text { has to be removed or not. If not it is equal to } 0 \text {. }\end{array}$ \\
\hline
\end{tabular}

In case of many $\mathrm{MN}$ ongoing sessions, sending multiple SIP re-INVITE messages may overload the radio interface. An alternative solution would be to include the information (UFA_Terminal_Config, UFA_Appli_Config, UFA_GW T IP address, and Proposed SDP) related to all sessions subject to handover in a single SIP NOTIFY [14] message, supposing the $\mathrm{MN}$ has subscribed to a handover event. However this would require complex treatment in the terminal which has to extract the content of NOTIFY message and send internally a SIP reINVITE message to the corresponding SIP UA (User Agent). A 
trade-off between radio load and terminal complexity shall be found. In our case, as shown in Figure 3, we choose to use SIP re-INVITE messages. When the terminal has many service sessions and in the usecases where UFA_Terminal_Config indicates that current IP address shall be deleted (for example the case of mono-interfaced terminal performing a hard $\mathrm{HO}$ ) the application of the content of the first received SIP reINVITE message will delete the current IP address and prevent from the reception of the other SIP re-INVITE messages related to the other sessions. To avoid this, we added a third SIP header called UFA_Terminal_Synchronisation that specifies to the terminal the number of SIP re-INVITE messages it has to wait for before executing UFA Terminal Config. To support $\mathrm{HO}$, the terminal implements the architecture shown in Figure 4. An "All-Layer Configuration Module" intercepts SIP reINVITE messages, extracts UFA_Terminal_Config and UFA Terminal Synchronisation and relays the SIP re-INVITE message to SIP UA. It then enforces L2 and L3 HOs according to UFA_Terminal_Synchronisation.

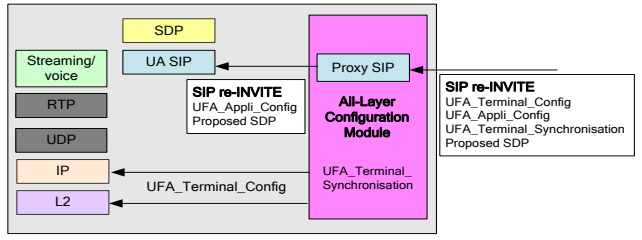

Figure 4. MN architecture

To avoid packet loss during HO execution, UFA_GW_S can transfer packets received from $\mathrm{CN}$ to UFA_GW_T that buffers them. After $\mathrm{HO}$, the MN sends a SIP re-INVITE message (8) to UFA_GW_T, which activates the MN context and begins data sending.

\section{UFA PRoOF OF CONCEPT AND PERFormance RESUlts}

To prove UFA concept and check the applicability of the above mobility procedure, we implemented UFA on a testbed. The testbed is given by the Figure 5. It consists of four computers. A desktop running Fedora Core 7 with kernel version 2.6.23 is used as UFA_CN. Two other desktops running Ubuntu 8.10 with kernel version 26.28.2 act as two UFA_GWs, they are equipped with $3 \mathrm{com}$ Wireless a/b/g PCi adapters. A laptop running Ubuntu 8.10 with kernel version 2.6.28.2 is the $\mathrm{MN}$, it has a wireless Netgear $\mathrm{a} / \mathrm{b} / \mathrm{g}$ card. The testbed is based on IPv6.

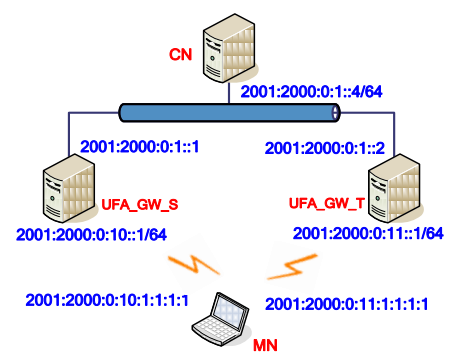

Figure 5. UFA testbed
We implemented the B2BUA, the UFA Terminal Config and UFA Appli_Config headers and all UFA HO messages excluding messages 1 and 2. Data transfer between the GWs and data buffering in the UFA_GW_T during HO are let for a further step of the testbed development. After session establishment, $\mathrm{CN}$ sends a streaming voice file to $\mathrm{MN}$. We evaluated UFA performance regarding messages lengths and $\mathrm{HO}$ delays. The length of messages $5 \mathrm{~A}, 6 \mathrm{~A}, 7 \mathrm{~A}, 5,6,7$ are respectively $1229,544,494,929,535,485$ bytes. -We considered the hard $\mathrm{HO}$ case as it is the most restricting one. The HO delay is measured at the application level (Appli_HO_Delay), it represents the delay between the last received data (D) packet before $\mathrm{HO}$ and the first received data packet after HO, its average value Appli_HO_Delay(UFA) on the testbed is $100 \mathrm{~ms}$. To show the relative gain of our solution, we implemented the classic SIP-based mobility scheme (reference case, $(\mathrm{REF})$ ) shown in Figure 6. The measured Appli_HO_Delay(REF) is $1258 \mathrm{~ms}$ in average for a configuration with 50ms-periodic Router Advertisements (RA) [15] and an activated Duplicate Address Detection (DAD) [15] Appli_HO Delay(REF) is 12 times larger than Appli_HO_Delay(UFA). Figure 6 and Figure 7 show respectively the HO delay components for (REF) and (UFA) cases. Table 3 defines these components and gives their measured values. Appli_HO Delay(REF) includes at least the delay components due to L 2 and L3 HO and IP path update signaling. During tests, we observed for (REF) case that MN does not consider the first received RA, which explains the obtained $130 \mathrm{~ms}$ for D2 instead of $50 \mathrm{~ms}$ maximum.

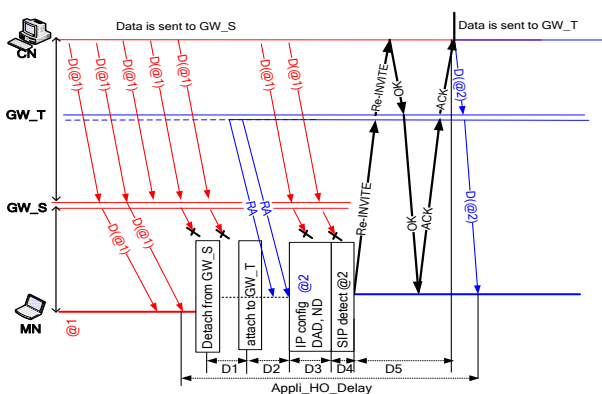

Figure 6. Reference (REF) case HO delays

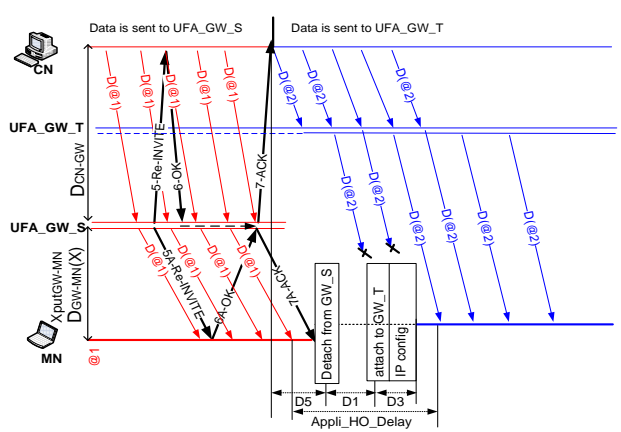

Figure 7. UFA HO delays 
Comparing (UFA) and (REF) cases: D1 is the same for (UFA) and (REF); D2 in (UFA) does not exist since the MN address is proactively determined before $\mathrm{HO}$ execution order; $\mathrm{D} 3$ in (UFA) is very low given the fact that DAD has been performed proactively by UFA GW_T and L2 and L3 configurations are simultaneously performed; D4 does not exit in (UFA) as SIP signaling is sent by UFA_GW_S. D5 is to due to SIP signaling delay; in (UFA) it corresponds to the time difference between the reception of message 7 on $\mathrm{CN}$ and the reception of message 7A on MN. Despite the very low delay links considered for these measurements, D5 is not null for (REF). This is explained by the fact that when GW_T receives the $O K$ message from $\mathrm{CN}$ towards $\mathrm{MN}$, it needs to perform $\mathrm{ND}$ (Neighbor Discovery) [15] towards MN to determine its MAC address. This does not occur in (UFA) for first data received by the UFA_GW_T towards $\mathrm{MN}$ as we configured the UFA GW T neighbor table during the HO preparation phase Note that Appli_HO_Delay(REF) and Appli_HO_Delay(UFA) are slightly different from (D1+D2+D3+D4+D5) and (D1+D3) respectively as voice packets are not sent continuously bu spaced by $20 \mathrm{~ms}$.

Table 3. Appli_HO_Delay components

\begin{tabular}{|l|l|l|} 
Appli_HO_Delay components & REF & UFA \\
\hline
\end{tabular}

\begin{tabular}{|l|l|l|}
$\mathrm{D}_{1}$ : the time necessary for $\mathrm{MN}$ to perform $\mathrm{L} 2$ & $\mathbf{6 0}$ & $\mathbf{6 0}$
\end{tabular}

HO (ms)

$\mathrm{D}_{2}$ : the time necessary to receive Router 13 Advertisement (ms)

\begin{tabular}{|l|l|l}
$\mathrm{D}_{3}$ : the time necessary for MN to be able to use & $\mathbf{1 0 0 0}$ & $\mathbf{2 0}$
\end{tabular}

its interface at IP level, it is due to DAD

procedure, interface configuration and ND (ms)

$\mathrm{D}_{4}$ : the time necessary for MN to detect IP HO

at SIP level and build the corresponding re-

INVITE message (ms)

D5: the time necessary to exchange SIP

signaling messages (SIP re-INVITE message,

$\operatorname{SIP} O K, \operatorname{SIP} A C K)(\mathrm{ms})$

We also measured Appli_HO_Delay(UFA) for different network configurations by varying $\mathrm{D}_{\mathrm{CN}-\mathrm{GW}}$ and $\mathrm{D}_{\mathrm{GW}-\mathrm{MN}}(\mathrm{X})$ parameters. $\mathrm{D}_{\mathrm{CN}-\mathrm{GW}}$ is the propagation delay on the $\mathrm{CN}-\mathrm{GW}$ link. We assumed that packets on this link are only impacted by this delay and not by the link throughput. $\mathrm{D}_{\mathrm{GW}-\mathrm{MN}}(\mathrm{X})$ is the transmission delay of a packet $\mathrm{X}$ over the MN-GW wireless link. It is due to the throughput $\mathrm{Xput}_{\mathrm{GW}-\mathrm{MN}}$ of this link. $\mathrm{D}_{\mathrm{CN}-\mathrm{GW}}$ and $\mathrm{Xput}_{\mathrm{GW}-\mathrm{MN}}$ are varied on the testbed thanks to Linux $t c$ qdisc commands. According to Figure 7, the content of SIP reINVITE messages are considered by $\mathrm{CN}$ and $\mathrm{MN}$ only after the reception of message $7(A C K)$ and $7 \mathrm{~A}(A C K)$ respectively. When $\left(\mathrm{D}_{\mathrm{GW}-\mathrm{MN}}(5 \mathrm{~A}+6 \mathrm{~A}+7 \mathrm{~A}) \gg 3 * \mathrm{D}_{\mathrm{CN}-\mathrm{GW}}\right)$, the $\mathrm{CN}$ may begin data transmission towards Add IP Addr very earlier than the $\mathrm{MN}$ attachment to UFA GW T causing thus a considerable data loss. To optimize the solution performance, UFA_GW_S does not send $A C K$ message (7) to $\mathrm{CN}$ before the reception of $O K$ message (6A) from $\mathrm{MN}$ (see Figure 7), this reduces the time difference between message 7 and 7A reception on $\mathrm{CN}$ and $\mathrm{MN}$ to $\left|\mathrm{D}_{\mathrm{GW}-\mathrm{MN}}(7 \mathrm{~A})-\mathrm{D}_{\mathrm{CN}-\mathrm{GW}}\right|$. When $\left(\mathrm{D}_{\mathrm{GW}}\right.$ $\left.\mathrm{MN}(5 \mathrm{~A}+6 \mathrm{~A}+7 \mathrm{~A}) \quad \ll \quad 3 * \mathrm{D}_{\mathrm{CN}-\mathrm{GW}}\right)$, to avoid losing the connectivity with MN in hard HO situation, UFA GW S does not delay the sending of message 7A to $\mathrm{MN}$ until the reception oof message 6 from CN. Given these assumptions, Appli_HO_Delay(UFA) can be calculated as follows

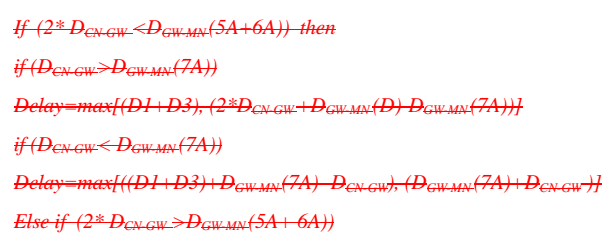

Figure 8Figure 8 shows Appli_HO_Delay(UFA) measured on the testbed for different radio bitrates $\left(\right.$ Xput $\left._{\mathrm{GW}-\mathrm{MN}}\right)$. The obtained values are consistent with the analytical values ealeulated with the above equations as shown also on the same figure-We observe that Appli_HO Delay(UFA) with highdelay links remains inferior to Appli_HO_Delay(REF) with 0 ms-delay links. The irregularity of the obtained curves in (UFA) is due to the asynchronous consideration of messages 7 and $7 \mathrm{~A}$ on $\mathrm{CN}$ and $\mathrm{MN}$ sides which occur when $\mathrm{D}_{\mathrm{GW}-\mathrm{MN}}(\mathrm{X})$ is very different from on $\mathrm{D}_{\mathrm{CN}-\mathrm{GW}}$. For $\mathrm{D}_{\mathrm{CN}-\mathrm{GW}}=10 \mathrm{~ms}$ and for a high radio bitrate, $\mathrm{D}_{\mathrm{GW}-\mathrm{MN}}(\mathrm{X})$ is small and inferior to $\mathrm{DCN}$ GW, which gives a minimal Appli_HO_Delay(UFA) close to (D1+D3).
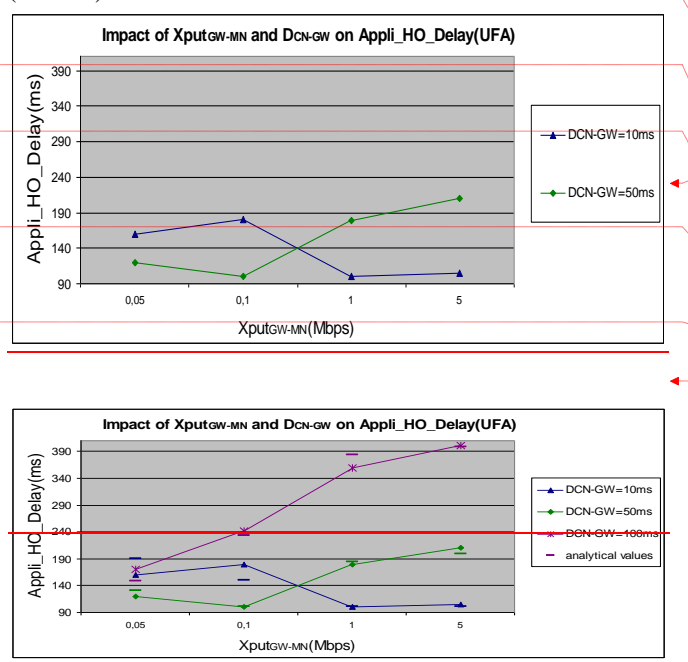

Figure 8. Appli_HO_Delay(UFA) measured on the testbed

\section{CONCLUSION}

UFA is a new mobile network architecture specified with the goal of reducing scalability issues expected for IMS and current mobile networks in case of a high number of users consuming high bitrate services. The main principle of UFA is to distribute IMS and mobile network functions into the base stations. UFA establishment and mobility procedures integrate QoS and are network-controlled allowing an optimal use of network resources. UFA implementation on a testbed has
Mis en forme : Corps de texte, Retrait : Gauche : $0 \mathrm{~cm}$, Espace Avan $: 6 \mathrm{pt}$

Mis en forme : Corps de texte Espace Avant : 6 pt

Mis en forme : Espace Avant : $0 \mathrm{pt}$

Mis en forme : Vérifier l'orthographe et la grammaire

Code de champ modifié

Mis en forme : Police : $10 \mathrm{pt}$

Mis en forme : Non Exposant/ Indice

Mis en forme : Non Exposant/ Indice

Mis en forme : Non Exposant/ Indice

Mis en forme : Non Exposant/ Indice

Mis en forme : Police : $10 \mathrm{pt}$

Mis en forme: Police : Non Italique

Mis en forme : Police : $10 \mathrm{pt}$

Mis en forme : Retrait : Première ligne : $0 \mathrm{~cm}$, Espace Avant : 0 pt

Mis en forme : Police :10 pt

Mis en forme : Police : $10 \mathrm{pt}$

Mis en forme : Police : $10 \mathrm{pt}$

Mis en forme : Police :10 pt

Mis en forme : Retrait : Première

ligne: $0 \mathrm{~cm}$

Mis en forme : Espace Avant : $6 \mathrm{pt}$ Après : 3 pt 
enabled the validation of its concept and allowed to measure the $\mathrm{HO}$ delay for different network delays. The minimum $\mathrm{HO}$ value is $100 \mathrm{~ms}$ for hard HO. This shows that UFA respects the requirements of the most restricting applications. Next work will also concern the interest of UFA and SIP use for non-SIP based applications.

\section{NOTICE}

UFA is being studied in P1857 Eurescom project and interests France Telecom, Portugal Telecom, Mobile Innovation Centre Hungary.

\section{REFERENCES}

11] 3GPP TS 23.228, "IP Multimedia Subsystem (IMS)".

2] K. Daoud, P.Herbelin, Noel Crespi, "UFA: An Ultra Flat Architecture for High Bitrate Services in Mobile Networks", PIMRC 2008.

3GPP TS 23.060, "GPRS Service description", release 9.

[4] 3GPP TR 25.999, "High Speed Packet Access (HSPA) evolution"

[5] 3GPP TS 23.402, "3GPP System Architecture Evolution: Architecture Enhancements for non-3GPP accesses".

[6] 3GPP TS 36.300, "Evolved Universal Terrestrial Radio Access (EUTRA) and Evolved Universal Terrestrial Radio Access Network (EUTRA) and Evolved Univer

7] IETF RFC 5213: "Proxy Mobile IPv6".

8] IETF RFC 3261, " SIP: Session Initiation Protocol".

[9] 3GPP TS 23.203, "Policy Control and Charging architecture".

[10] K. Daoud, P.Herbelin, Noel Crespi, "One-Node Based Mobile architecture for better QoS control", IFIP Wireless days 2008.

[11] P. Agrawal, et.al, "IP Multimedia Subsystems in 3GPP \& 3GPP2 P. Agrawal, et.al, "IP Multimedia Subsystems in 3GPP \& 3GP
Scalability issues", IEEE Communications Magazine, January, 2008.

12] IETF RFC 2327, " SDP: Session Description Protocol ".

13] IETF RFC 3515, " The Session Initiation Protocol (SIP) Refer Method".

14] IETF RFC 3265, "Session Initiation Protocol (SIP)-Specific Even Notification"

[15] IETF RFC 2461, "Neighbor Discovery for IP Version 6 (IPv6)" 